\title{
An improved regularity criterion for the Navier-Stokes equations in terms of one directional derivative of the velocity field
}

\author{
Zujin Zhang ${ }^{1}$
}

Received: 4 December 2015 / Revised: 24 December 2016 / Accepted: 28 December 2016 /

Published online: 4 January 2017

(C) The Author(s) 2017. This article is published with open access at Springerlink.com

\begin{abstract}
In this paper, we establish a new multiplicative Sobolev inequality. As applications, we refine and extend the results in Kukavica and Ziane (J Math Phys 48:065203, 2007) and Cao (Discrete Contin Dyn Syst 26:1141-1151, 2010) simultaneously.
\end{abstract}

Keywords Regularity criteria · Navier-Stokes equations · Multiplicative Sobolev inequality

Mathematics Subject Classification 35B65 - 35Q30 · 76D03

\section{Introduction}

The homogeneous incompressible fluid flow is governed by the following NavierStokes equations (NSE):

$$
\left\{\begin{array}{l}
\partial_{t} \boldsymbol{u}+(\boldsymbol{u} \cdot \nabla) \boldsymbol{u}-\Delta \boldsymbol{u}+\nabla \pi=\mathbf{0} \\
\nabla \cdot \boldsymbol{u}=0 \\
\boldsymbol{u}(0)=\boldsymbol{u}_{0}
\end{array}\right.
$$

where $\boldsymbol{u}=\left(u_{1}, u_{2}, u_{3}\right)$ is the fluid velocity field, $\pi$ is a scalar pressure, $\boldsymbol{u}_{0}$ is the prescribed initial velocity field satisfying the compatibility condition $\nabla \cdot \boldsymbol{u}_{0}=0$, and

\section{Communicated by Neil Trudinger.}

Zujin Zhang

zhangzujin361@163.com

1 School of Mathematics and Computer Sciences, Gannan Normal University, Ganzhou 341000, Jiangxi, People's Republic of China 


$$
\partial_{t} \boldsymbol{u}=\frac{\partial \boldsymbol{u}}{\partial t}, \quad \partial_{i}=\frac{\partial}{\partial x_{i}}, \quad(\boldsymbol{u} \cdot \nabla)=\sum_{i=1}^{3} u_{i} \partial_{i} .
$$

The global existence of a weak solution to the evolutionary NSE (1) has been long established by Leray [19] and Hopf [9]; however, the issue of its regularity and uniqueness remains open up to now. Pioneered by Serrin [26], we began studying the regularity criterion for the NSE (1); that is, to find some sufficient condition to ensure the smoothness of the solution. The classical Prodi-Serrin conditions (see $[8,24,26]$ ) says that if

$$
\boldsymbol{u} \in L^{p}\left(0, T ; L^{q}\left(\mathbb{R}^{3}\right)\right), \quad \frac{2}{p}+\frac{3}{q}=1, \quad 3 \leq q \leq \infty,
$$

then the solution is regular on $(0, T)$.

This was be generalized by Beirão da Veiga [1] by considering the velocity gradient or vorticity,

$$
\nabla \boldsymbol{u} \in L^{p}\left(0, T ; L^{q}\left(\mathbb{R}^{3}\right)\right), \quad \frac{2}{p}+\frac{3}{q}=2, \quad \frac{3}{2} \leq q \leq \infty .
$$

Notice that the case $q \in\left[\frac{3}{2}, 3\right)$ follows directly from (2) and the Sobolev inequality.

In view of the divergence-free condition $\nabla \cdot \boldsymbol{u}=0$, it is natural to ask whether or not we can reduce (2) and (3) to its partial components. One way is to consider regularity criteria involving only one velocity component, which were done in $[3,11,16,20,34$, 36]. Another way is to study the possible components reduction of $\nabla \boldsymbol{u}$ to $\nabla u_{3}$, see $[16,23,27,35,36]$; or to $\partial_{3} \boldsymbol{u}$, see $[2,17,21,22]$. In [22], Penel-Pokorný showed that if

$$
\partial_{3} \boldsymbol{u} \in L^{p}\left(0, T ; L^{q}\left(\mathbb{R}^{3}\right)\right), \quad \frac{2}{p}+\frac{3}{q}=\frac{3}{2}, \quad 2 \leq q \leq \infty,
$$

then the solution is smooth. This was improved by Kukavica-Ziane [17] to be

$$
\partial_{3} \boldsymbol{u} \in L^{p}\left(0, T ; L^{q}\left(\mathbb{R}^{3}\right)\right), \quad \frac{2}{p}+\frac{3}{q}=2, \quad \frac{9}{4} \leq q \leq 3 .
$$

Notice that the range of $q$ is not of full range $\left(\frac{3}{2}, \infty\right]$. The reason is that in [17], the estimate of $I_{3}$ needs to be reconciled with the the estimate of $K$. Furthermore, this method was adjusted by Penel-Pokorný [21] to get an anisotropic criterion. For readers interested in this topic, please refer to [12-15,30] for recent progresses on regularity criteria of the MHD equations, which contains system (1) as a subsystem.

Later on, Cao [2] employed multiplicative Sobolev inequalities

$$
1 \leq q<\infty \Rightarrow\|f\|_{L^{3 q}} \leq C\left\|\partial_{1} f\right\|_{L^{2}}^{\frac{1}{3}}\left\|\partial_{2} f\right\|_{L^{2}}^{\frac{1}{3}}\left\|\partial_{3} f\right\|_{L^{q}}^{\frac{1}{3}}
$$


and

$$
1 \leq q<\infty \Rightarrow\|f\|_{L^{5 q}} \leq C\left\|\partial_{1}\left(f^{2}\right)\right\|_{L^{2}}^{\frac{1}{5}}\left\|\partial_{2}\left(f^{2}\right)\right\|_{L^{2}}^{\frac{1}{5}}\left\|\partial_{3} f\right\|_{L^{q}}^{\frac{1}{5}}
$$

to get the following extended regularity condition ${ }^{1}$

$$
\partial_{3} \boldsymbol{u} \in L^{p}\left(0, T ; L^{q}\left(\mathbb{R}^{3}\right)\right), \quad \frac{2}{p}+\frac{3}{q}=2, \quad \frac{27}{16} \leq q \leq \frac{5}{2} .
$$

Notice that the lower and upper bounds of $q$ in (8) both are less than those in (5) respectively. Consequently, our best knowledge in this direction is the following sufficient condition

$$
\partial_{3} \boldsymbol{u} \in L^{p}\left(0, T ; L^{q}\left(\mathbb{R}^{3}\right)\right), \quad \frac{2}{p}+\frac{3}{q}=2, \quad \frac{27}{16} \leq q \leq 3 .
$$

Some of them was proved in [17], while other parts could only be seen [2].

In this paper, we shall further generalize (7), and improve (5) and (8) simultaneously. We will show that the condition

$$
\partial_{3} \boldsymbol{u} \in L^{p}\left(0, T ; L^{q}\left(\mathbb{R}^{3}\right)\right), \quad \frac{2}{p}+\frac{3}{q}=2, \quad \frac{3 \sqrt{37}}{4}-3 \leq q \leq 3
$$

could ensure the regularity of the solution. Noting

$$
\frac{3 \sqrt{37}}{4} \approx 1.56207<1.6875=\frac{27}{16}
$$

we are much closer to the end point $\frac{3}{2}$.

Before stating the precise result, let us recall the weak formulation of (1), see $[7,18,25,28]$ for instance.

Definition 1 Let $\boldsymbol{u}_{0} \in L^{2}\left(\mathbb{R}^{3}\right)$ with $\nabla \cdot \boldsymbol{u}_{0}=0, T>0$. A measurable $\mathbb{R}^{3}$-valued function $\boldsymbol{u}$ defined in $[0, T] \times \mathbb{R}^{3}$ is said to be a weak solution to (1) if

1. $\boldsymbol{u} \in L^{\infty}\left(0, T ; L^{2}\left(\mathbb{R}^{3}\right) \cap L^{2}\left(0, T ; H^{1}\left(\mathbb{R}^{3}\right)\right)\right.$;

2. (1) 1 and (1) 2 hold in the sense of distributions, i.e.,

$$
\int_{0}^{t} \int_{\mathbb{R}^{3}} \boldsymbol{u} \cdot\left[\partial_{t} \boldsymbol{\phi}+(\boldsymbol{u} \cdot \nabla) \boldsymbol{\phi}\right] \mathrm{d} x \mathrm{~d} s+\int_{\mathbb{R}^{3}} \boldsymbol{u}_{0} \cdot \boldsymbol{\phi}(0) \mathrm{d} x=\int_{0}^{T} \int_{\mathbb{R}^{3}} \nabla \boldsymbol{u}: \nabla \boldsymbol{\phi} \mathrm{d} x \mathrm{~d} t,
$$

\footnotetext{
1 In [2, Theorem 2.1], the author claims that (8) is valid for all $q \in[27 / 16, \infty]$; however, only the case $q \in[27 / 16,5 / 2]$ could be verified in his paper, see the inequality just before (32).
} 
for each $\boldsymbol{\phi} \in C_{c}^{\infty}\left([0, T) \times \mathbb{R}^{3}\right)$ with $\nabla \cdot \boldsymbol{\phi}=0$, where $A: B=\sum_{i, j=1}^{3} a_{i j} b_{i j}$ for $3 \times 3$ matrices $A=\left(a_{i j}\right), B=\left(b_{i j}\right)$, and

$$
\int_{0}^{T} \int_{\mathbb{R}^{3}} \boldsymbol{u} \cdot \nabla \psi \mathrm{d} x \mathrm{~d} t=0
$$

for each $\psi \in C_{c}^{\infty}\left(\mathbb{R}^{3} \times[0, T)\right)$;

3. the energy inequality, that is,

$$
\|\boldsymbol{u}(t)\|_{L^{2}}^{2}+2 \int_{0}^{t}\|\nabla \boldsymbol{u}(s)\|_{L^{2}}^{2} \mathrm{~d} s \leq\left\|\boldsymbol{u}_{0}\right\|_{L^{2}}^{2}, \quad 0 \leq t \leq T .
$$

Now, our main result reads

Theorem 2 Let $\boldsymbol{u}_{0} \in L^{2}\left(\mathbb{R}^{3}\right)$ with $\nabla \cdot \boldsymbol{u}_{0}=0, T>0$. Assume that $\boldsymbol{u}$ is a weak solution to (1) on $[0, T]$ with initial data $\boldsymbol{u}_{0}$. If

$$
\partial_{3} \boldsymbol{u} \in L^{p}\left(0, T ; L^{q}\left(\mathbb{R}^{3}\right)\right), \quad \frac{2}{p}+\frac{3}{q}=2, \quad \frac{3 \sqrt{37}}{4}-3 \leq q \leq 3,
$$

then the solution $\boldsymbol{u}$ is smooth in $(0, T] \times \mathbb{R}^{3}$.

The proof of Theorem 2 will be given in Sect. 2. Before doing that, let us state our notations used throughout the paper, and prove a multiplicative Sobolev inequality.

For simplicity of presentation, we do not distinguish between the spaces $X$ and their $N$-dimensional vector analogs $X^{N}$ (e.g., $N=3$ for $\boldsymbol{u} \in L^{2}\left(\mathbb{R}^{3}\right), N=9$ for $\left.\nabla \boldsymbol{u} \in L^{2}\left(\mathbb{R}^{3}\right)\right)$; however, all vector- and tensor-valued functions are printed boldfaced. A constant $C$ may change from line to line, depending only on the initial data or the norms that we have controlled. We denote by

$$
\boldsymbol{u}_{h}=\left(u_{1}, u_{2}\right), \quad \nabla_{h}=\left(\partial_{1}, \partial_{2}\right), \quad \triangle_{h}=\partial_{1} \partial_{1}+\partial_{2} \partial_{2} .
$$

Generalizing (7) in [2], we have the following

Lemma 3 For each $1 \leq q<\infty, 0<\lambda<\infty$, there exists some constant $C$ such that for each $f \in C_{c}^{\infty}\left(\mathbb{R}^{3}\right)$,

$$
\|f\|_{L^{(2 \lambda+1) q}} \leq C\left\|\partial_{i} f\right\|_{L^{q}}^{\frac{1}{2 \lambda+1}}\left\|\partial_{j}\left(|f|^{\lambda}\right)\right\|_{L^{2}}^{\frac{1}{2 \lambda+1}}\left\|\partial_{k}\left(|f|^{\lambda}\right)\right\|_{L^{2}}^{\frac{1}{2 \lambda+1}}
$$

where $\{i, j, k\}$ is a permutation of $\{1,2,3\}$. 
Proof By Newton-Leibniz formula, we have

$$
\begin{aligned}
& |f|^{2 \lambda(q-1)+q} \leq C \int_{\mathbb{R}}|f|^{(2 \lambda+1)(q-1)}\left|\partial_{i} f\right| \mathrm{d} x_{i} \\
& |f|^{\lambda(q+1)+\frac{q}{2}}=\left(|f|^{\lambda}\right)^{q+1+\frac{q}{2 \lambda}} \leq C \int_{\mathbb{R}}|f|^{\frac{(2 \lambda+1) q}{2}}\left|\partial_{j}\left(|f|^{\lambda}\right)\right| \mathrm{d} x_{j}, \\
& |f|^{\lambda(q+1)+\frac{q}{2}} \leq C \int_{\mathbb{R}}|f|^{\frac{(2 \lambda+1) q}{2}}\left|\partial_{k}\left(|f|^{\lambda}\right)\right| \mathrm{d} x_{k} .
\end{aligned}
$$

Taking the sqrt of the multiplication of the above inequalities, we deduce

$$
\begin{aligned}
|f|^{(2 \lambda+1) q} \leq & {\left[\int_{\mathbb{R}}|f|^{(2 \lambda+1)(q-1)}\left|\partial_{i} f\right| \mathrm{d} x_{i}\right]^{\frac{1}{2}} } \\
& \times\left[\int_{\mathbb{R}}|f|^{\frac{(2 \lambda+1) q}{2}}\left|\partial_{j}\left(|f|^{\lambda}\right)\right| \mathrm{d} x_{j}\right]^{\frac{1}{2}} \cdot\left[\int_{\mathbb{R}}|f|^{\frac{(2 \lambda+1) q}{2}}\left|\partial_{k}\left(|f|^{\lambda}\right)\right| \mathrm{d} x_{k}\right]^{\frac{1}{2}}
\end{aligned}
$$

Integrating in the $x_{i}$ variable and applying Hölder inequality, we obtain

$$
\begin{aligned}
& \int_{\mathbb{R}}|f|^{(2 \lambda+1) q} \mathrm{~d} x_{i} \leq C\left[\int_{\mathbb{R}}|f|^{(2 \lambda+1)(q-1)}\left|\partial_{i} f\right| \mathrm{d} x_{i}\right]^{\frac{1}{2}} \\
& \cdot\left[\int_{\mathbb{R}^{2}}|f|^{\frac{(2 \lambda+1) q}{2}}\left|\partial_{j}\left(|f|^{\lambda}\right)\right| \mathrm{d} x_{i} \mathrm{~d} x_{j}\right]^{\frac{1}{2}} \cdot\left[\int_{\mathbb{R}^{2}}|f|^{\frac{(2 \lambda+1) q}{2}}\left|\partial_{k}\left(|f|^{\lambda}\right)\right| \mathrm{d} x_{i} \mathrm{~d} x_{k}\right]^{\frac{1}{2}}
\end{aligned}
$$

Successively, integrating in the $x_{j}$ and $x_{k}$ variables and applying Hölder inequality, we get

$$
\begin{aligned}
& \int_{\mathbb{R}^{3}}|f|^{(2 \lambda+1) q} \mathrm{~d} x \leq C\left[\int_{\mathbb{R}^{3}}|f|^{(2 \lambda+1)(q-1)}\left|\partial_{i} f\right| \mathrm{d} x\right]^{\frac{1}{2}} \\
& \cdot\left[\int_{\mathbb{R}^{3}}|f|^{\frac{(2 \lambda+1) q}{2}}\left|\partial_{j}\left(|f|^{\lambda}\right)\right| \mathrm{d} x\right]^{\frac{1}{2}} \cdot\left[\int_{\mathbb{R}^{3}}|f|^{\frac{(2 \lambda+1) q}{2}}\left|\partial_{k}\left(|f|^{\lambda}\right)\right| \mathrm{d} x\right]^{\frac{1}{2}} .
\end{aligned}
$$

Invoking Hölder inequality again, we find

$$
\begin{aligned}
\|f\|_{L^{(2 \lambda+1) q}}^{(2 \lambda+1) q} \leq C & {\left[\|f\|_{L^{(2 \lambda+1) q}}^{(2 \lambda+1)(q-1)}\left\|\partial_{i} f\right\|_{L^{q}}^{\frac{1}{2}}\right]^{\frac{1}{2}} } \\
& \cdot\left[\|f\|_{L^{(2 \lambda+1) q}}^{\frac{(2 \lambda+1) q}{2}}\left\|\partial_{j}\left(|f|^{\lambda}\right)\right\|_{L^{2}}\right]^{\frac{1}{2}}\left[\|f\|_{L^{(2 \lambda+1) q}}^{\frac{(2 \lambda+1) q}{2}}\left\|\partial_{k}\left(|f|^{\lambda}\right)\right\|_{L^{2}}\right]^{\frac{1}{2}} \\
\leq C & \|f\|_{L^{(2 \lambda+1) q}}^{(2 \lambda+1)\left(q-\frac{1}{2}\right)}\left\|\partial_{i} f\right\|_{L^{q}}^{\frac{1}{2}}\left\|\partial_{j}\left(|f|^{\lambda}\right)\right\|_{L^{2}}^{\frac{1}{2}}\left\|\partial_{k}\left(|f|^{\lambda}\right)\right\|_{L^{2}}^{\frac{1}{2}}
\end{aligned}
$$

Dividing both sides by $\|f\|_{L^{(2 \lambda+1) q}}^{(2 \lambda+1)\left(q-\frac{1}{2}\right)}$, we finished the proof of Lemma 3 . 


\section{Proof of Theorem 2}

In this section, we shall prove Theorem 2.

For any $\varepsilon \in(0, T)$, due to the fact that $\nabla \boldsymbol{u} \in L^{2}\left(0, T ; L^{2}\left(\mathbb{R}^{3}\right)\right)$, we may find a $\delta \in(0, \varepsilon)$, such that $\nabla \boldsymbol{u}(\delta) \in L^{2}\left(\mathbb{R}^{3}\right)$. Take this $\boldsymbol{u}(\delta)$ as initial data, there exists an $\tilde{\boldsymbol{u}} \in C\left(\left[\delta, \Gamma^{*}\right), H^{1}\left(\mathbb{R}^{3}\right)\right) \cap L^{2}\left(0, \Gamma^{*} ; H^{2}\left(\mathbb{R}^{3}\right)\right)$, where $\left[\delta, \Gamma^{*}\right)$ is the life span of the unique strong solution, see [28]. Moreover, $\tilde{\boldsymbol{u}} \in C^{\infty}\left(\mathbb{R}^{3} \times\left(\delta, \Gamma^{*}\right)\right)$. According to the uniqueness result, $\tilde{\boldsymbol{u}}=\boldsymbol{u}$ on $\left[\delta, \Gamma^{*}\right)$. If $\Gamma^{*} \geq T$, we have already that $\boldsymbol{u} \in$ $C^{\infty}\left(\mathbb{R}^{3} \times(0, T)\right)$, due to the arbitrariness of $\varepsilon \in(0, T)$. In case $\Gamma^{*}<T$, our strategy is to show that $\left\|\nabla \boldsymbol{u}_{h}(t)\right\|_{2}$ remains uniform bounded as $t \nearrow \Gamma^{*}$. By [33, Proposition 1.1], we have $\|\nabla \boldsymbol{u}(t)\|_{2}$ remains uniform bounded as $t \nearrow \Gamma^{*}$. The standard continuation argument then yields that $\left[\delta, \Gamma^{*}\right)$ could not be the maximal interval of existence of $\tilde{\boldsymbol{u}}$, and consequently $\Gamma^{*} \geq T$. This concludes the proof.

By (11), we may find a $\Gamma<\Gamma^{*}$ such that

$$
\|\nabla \boldsymbol{u}(\Gamma)\|_{L^{2}} \leq C, \quad\left(\int_{\Gamma}^{\Gamma^{*}}\left\|\partial_{3} \boldsymbol{u}\right\|_{L^{q}}^{\frac{2 q}{2 q-3}} \mathrm{~d} t\right)^{\frac{2 q-3}{2 q}}<\tilde{\varepsilon}
$$

where $0<\tilde{\varepsilon} \ll 1$ will be determined later on.

For convenience, we rewrite the NSE (1) as

$$
\begin{aligned}
& \partial_{t} \boldsymbol{u}_{h}+\left(\boldsymbol{u}_{h} \cdot \nabla\right) \boldsymbol{u}_{h}+u_{3} \partial_{3} \boldsymbol{u}_{h}-\triangle_{h} \boldsymbol{u}_{h}-\partial_{3} \partial_{3} \boldsymbol{u}_{h}+\nabla_{h} \pi=\mathbf{0}, \\
& \partial_{t} u_{3}+\left(\boldsymbol{u}_{h} \cdot \nabla\right) u_{3}+u_{3} \partial_{3} u_{3}-\triangle_{h} u_{3}-\partial_{3} \partial_{3} u_{3}+\partial_{3} \pi=0, \\
& \nabla_{h} \cdot \boldsymbol{u}_{h}+\partial_{3} u_{3}=0 .
\end{aligned}
$$

\section{$2.1 H^{1}$ estimate}

Taking the inner product of $(13)_{1}$ with $-\triangle \boldsymbol{u}_{h}$ and $(1)_{1}$ with $-\partial_{3} \partial_{3} \boldsymbol{u}$ in $L^{2}\left(\mathbb{R}^{3}\right)$ respectively, we obtain

$$
\begin{aligned}
& \frac{1}{2} \frac{\mathrm{d}}{\mathrm{d} t}\left[\left\|\nabla \boldsymbol{u}_{h}\right\|_{L^{2}}^{2}+\left\|\partial_{3} \boldsymbol{u}\right\|_{L^{2}}^{2}\right]+\left[\left\|\Delta \boldsymbol{u}_{h}\right\|_{L^{2}}^{2}+\left\|\nabla \partial_{3} \boldsymbol{u}\right\|_{L^{2}}^{2}\right] \\
& \quad=\int_{\mathbb{R}^{3}}\left[(\boldsymbol{u} \cdot \nabla) \boldsymbol{u}_{h}\right] \cdot \Delta \boldsymbol{u}_{h} \mathrm{~d} x+\int_{\mathbb{R}^{3}} \nabla_{h} \pi \cdot \Delta \boldsymbol{u}_{h} \mathrm{~d} x+\int_{\mathbb{R}^{3}}[(\boldsymbol{u} \cdot \nabla) \boldsymbol{u}] \cdot \partial_{3} \partial_{3} \boldsymbol{u} \mathrm{d} x \\
& \quad \equiv I_{1}+I_{2}+I_{3} .
\end{aligned}
$$

By [17, Lemma 2.2],

$$
\begin{aligned}
I_{1} & =\int_{\mathbb{R}^{3}}\left[\left(\boldsymbol{u}_{h} \cdot \nabla_{h}\right) \boldsymbol{u}_{h}\right] \cdot \Delta_{h} \boldsymbol{u}_{h} \mathrm{~d} x+\int_{\mathbb{R}^{3}} u_{3} \partial_{3} \boldsymbol{u}_{h} \cdot \Delta \boldsymbol{u}_{h} \mathrm{~d} x \\
& =\frac{1}{2} \int_{\mathbb{R}^{3}} \partial_{3} u_{3}\left|\nabla_{h} \boldsymbol{u}_{h}\right|^{2} \mathrm{~d} x-\int_{\mathbb{R}^{3}} \partial_{1} u_{1} \partial_{2} u_{2} \partial_{3} u_{3} \mathrm{~d} x+\int_{\mathbb{R}^{3}} \partial_{1} u_{2} \partial_{2} u_{1} \partial_{3} u_{3} \mathrm{~d} x
\end{aligned}
$$




$$
\begin{aligned}
& +\int_{\mathbb{R}^{3}} u_{3} \partial_{3} \boldsymbol{u}_{h} \cdot \Delta \boldsymbol{u}_{h} \mathrm{~d} x \\
\leq & C \int_{\mathbb{R}^{3}}\left|\partial_{3} \boldsymbol{u}\right| \cdot\left|\nabla_{h} \boldsymbol{u}_{h}\right|^{2} \mathrm{~d} x+\int_{\mathbb{R}^{3}}\left|u_{3}\right| \cdot\left|\partial_{3} \boldsymbol{u}\right| \cdot\left|\triangle \boldsymbol{u}_{h}\right| \mathrm{d} x .
\end{aligned}
$$

To simplify $I_{2}$, we take the divergence of $(1)_{1}$ to get

$$
\begin{aligned}
-\triangle \pi= & \sum_{i, j=1}^{3} \partial_{i}\left(u_{j} \partial_{j} u_{i}\right) \\
= & \left.\sum_{i, j=1}^{2} \partial_{i} u_{j} \partial_{j} u_{i} \quad \text { (the case: } i \neq 3, j \neq 3\right) \\
& +\sum_{j=1}^{2} \partial_{3} u_{j} \partial_{j} u_{3}+u_{j} \partial_{j} \partial_{3} u_{3} \quad(\text { the case: } i=3, j \neq 3) \\
& +\sum_{i=1}^{3} \partial_{i} u_{3} \partial_{3} u_{i} \quad(\text { the case: } j=3)
\end{aligned}
$$

and thus

$$
\begin{aligned}
I_{2}= & -\int_{\mathbb{R}^{3}} \Delta \pi \cdot \nabla_{h} \boldsymbol{u}_{h} \mathrm{~d} x=\int_{\mathbb{R}^{3}} \triangle \pi \cdot \partial_{3} u_{3} \mathrm{~d} x \\
= & \sum_{i, j=1}^{2} \int_{\mathbb{R}^{3}} \partial_{i} u_{j} \partial_{j} u_{i} \partial_{3} u_{3} \mathrm{~d} x+\sum_{j=1}^{2} \int_{\mathbb{R}^{3}}\left(\partial_{3} u_{j} \partial_{j} u_{3}+u_{j} \partial_{j} \partial_{3} u_{3}\right) \partial_{3} u_{3} \mathrm{~d} x \\
& +\sum_{i=1}^{3} \int_{\mathbb{R}^{3}} \partial_{i} u_{3} \partial_{3} u_{i} \partial_{3} u_{3} \mathrm{~d} x \\
\leq & \int_{\mathbb{R}^{3}}\left|\partial_{3} u_{3}\right| \cdot\left|\nabla_{h} \boldsymbol{u}_{h}\right|^{2} \mathrm{~d} x-\sum_{j=1}^{2} \int_{\mathbb{R}^{3}} u_{3}\left(\partial_{3} \partial_{j} u_{j} \partial_{3} u_{3}+\partial_{3} u_{j} \partial_{j} \partial_{3} u_{3}\right) \mathrm{d} x \\
& +\frac{1}{2} \int_{\mathbb{R}^{3}}\left(\partial_{3} u_{3}\right)^{2} \mathrm{~d} x-\sum_{i=1}^{3} \int_{\mathbb{R}^{3}} u_{3} \partial_{3} u_{i} \partial_{i} \partial_{3} u_{3} \mathrm{~d} x \\
\leq & C \int_{\mathbb{R}^{3}}\left|\partial_{3} u_{3}\right| \cdot\left|\nabla_{h} \boldsymbol{u}_{h}\right|^{2} \mathrm{~d} x+\int_{\mathbb{R}^{3}}\left|u_{3}\right| \cdot\left|\partial_{3} \boldsymbol{u}\right| \cdot\left|\nabla \partial_{3} \boldsymbol{u}\right| \mathrm{d} x .
\end{aligned}
$$

Finally, integrating by parts yields

$$
\begin{aligned}
I_{3} & =-\int_{\mathbb{R}^{3}}\left[\left(\partial_{3} \boldsymbol{u} \cdot \nabla\right) \boldsymbol{u}\right] \cdot \partial_{3} \boldsymbol{u} \mathrm{d} x \\
& =-\int_{\mathbb{R}^{3}}\left[\left(\partial_{3} \boldsymbol{u}_{h} \cdot \nabla_{h}\right) \boldsymbol{u}_{h}\right] \cdot \partial_{3} \boldsymbol{u}_{h}+\left[\left(\partial_{3} \boldsymbol{u}_{h} \cdot \nabla_{h}\right) u_{3}\right] \cdot \partial_{3} u_{3}+\partial_{3} u_{3} \partial_{3} \boldsymbol{u} \cdot \partial_{3} \boldsymbol{u} \mathrm{d} x \\
& \leq C \int_{\mathbb{R}^{3}}\left|\partial_{3} \boldsymbol{u}\right| \cdot\left|\nabla \boldsymbol{u}_{h}\right|^{2} \mathrm{~d} x+C \int_{\mathbb{R}^{3}}\left|u_{3}\right| \cdot\left|\partial_{3} \boldsymbol{u}\right| \cdot\left|\nabla \partial_{3} \boldsymbol{u}\right| \mathrm{d} x
\end{aligned}
$$


Gathering (15), (17) and (18) into (14), we deduce

$$
\begin{aligned}
& \frac{1}{2} \frac{\mathrm{d}}{\mathrm{d} t}\left[\left\|\nabla \boldsymbol{u}_{h}\right\|_{L^{2}}^{2}+\left\|\partial_{3} \boldsymbol{u}\right\|_{L^{2}}^{2}\right]+\left[\left\|\Delta \boldsymbol{u}_{h}\right\|_{L^{2}}^{2}+\left\|\nabla \partial_{3} \boldsymbol{u}\right\|_{L^{2}}^{2}\right] \\
& \quad \leq C \int_{\mathbb{R}^{3}}\left|\partial_{3} \boldsymbol{u}\right| \cdot\left|\nabla \boldsymbol{u}_{h}\right|^{2} \mathrm{~d} x+C \int_{\mathbb{R}^{3}}\left|u_{3}\right| \cdot\left|\partial_{3} \boldsymbol{u}\right| \cdot\left(\left|\triangle \boldsymbol{u}_{h}\right|+\left|\nabla \partial_{3} \boldsymbol{u}\right|\right) \mathrm{d} x \\
& \quad \equiv J_{1}+J_{2}+J_{3} .
\end{aligned}
$$

By Hölder and Gagliardo-Nirenberg inequalities,

$$
\begin{aligned}
J_{1} & \leq C\left\|\partial_{3} \boldsymbol{u}\right\|_{L^{q}}\left\|\nabla \boldsymbol{u}_{h}\right\|_{L^{\frac{2 q}{q-1}}}^{2} \\
& \leq C\left\|\partial_{3} \boldsymbol{u}\right\|_{L^{q}}\left\|\nabla \boldsymbol{u}_{h}\right\|_{L^{\frac{2 q-3}{q}}}^{\frac{2 q-3}{2^{2}}}\left\|\nabla^{2} \boldsymbol{u}_{h}\right\|_{L^{2}}^{\frac{3}{q}} \\
& \leq C\left\|\partial_{3} \boldsymbol{u}\right\|_{L^{q}}\left\|\nabla \boldsymbol{u}_{h}\right\|_{L^{2}}^{\frac{2 q-3}{q}}\left\|\Delta \boldsymbol{u}_{h}\right\|_{L^{2}}^{\frac{3}{q}}
\end{aligned}
$$

For $J_{2}$, we first use Hölder inequality with

$$
1 \leq a, b \leq \infty, \quad \frac{1}{a}+\frac{1}{b}=\frac{1}{2}
$$

to estimate as

$$
J_{2} \leq C\left\|u_{3}\right\|_{L^{a}}\left\|\partial_{3} \boldsymbol{u}\right\|_{L^{b}}\left\|\left(\Delta \boldsymbol{u}_{h}, \nabla \partial_{3} \boldsymbol{u}\right)\right\|_{L^{2}},
$$

then invoke the interpolation and Gagliardo-Nirenberg inequalities to bound as

$$
J_{2} \leq C\left\|u_{3}\right\|_{L^{2 \lambda}}^{1-\vartheta_{1}}\left\|u_{3}\right\|_{L^{(2 \lambda+1) q}}^{\vartheta_{1}} \cdot\left\|\partial_{3} \boldsymbol{u}\right\|_{L^{q}}^{1-\vartheta_{2}}\left\|\nabla \partial_{3} \boldsymbol{u}\right\|_{L^{2}}^{\vartheta_{2}} \cdot\left\|\left(\Delta \boldsymbol{u}_{h}, \nabla \partial_{3} \boldsymbol{u}\right)\right\|_{L^{2}},
$$

where

$$
\frac{1}{a}=\frac{1-\vartheta_{1}}{2 \lambda}+\frac{\vartheta_{1}}{(2 \lambda+1) q}, \quad \frac{1}{b}=\frac{1-\vartheta_{2}}{q}+\vartheta_{2}\left(-\frac{1}{3}+\frac{1}{2}\right), \quad 0 \leq \vartheta_{1}, \vartheta_{2} \leq 1,
$$

and $\lambda \geq \frac{3}{2}$ will be specified later on.

By Lemma 3,

$$
\begin{aligned}
J_{2} & \leq C\left\|\left|u_{3}\right|^{\lambda}\right\|_{L^{2}}^{\frac{1-\vartheta_{1}}{\lambda}} \cdot\left\|\nabla_{h}\left(\left|u_{3}\right|^{\lambda}\right)\right\|_{L^{2}}^{\frac{2 \vartheta_{1}}{2 \lambda+1}}\left\|\partial_{3} u_{3}\right\|_{L^{q}}^{\frac{\vartheta_{1}}{2 \lambda+1}} \cdot\left\|\partial_{3} \boldsymbol{u}\right\|_{L^{q}}^{1-\vartheta_{2}}\left\|\left(\Delta \boldsymbol{u}_{h}, \nabla \partial_{3} \boldsymbol{u}\right)\right\|_{L^{2}}^{1+\vartheta_{2}} \\
& \leq C\left\|\left|u_{3}\right|^{\lambda}\right\|_{L^{2}}^{\frac{1-\vartheta_{1}}{\lambda}}\left\|\nabla_{h}\left(\left|u_{3}\right|^{\lambda}\right)\right\|_{L^{2}}^{\frac{2 \vartheta_{1}}{2 \lambda+1}}\left\|\partial_{3} \boldsymbol{u}\right\|_{L^{q}}^{\frac{\vartheta_{1}}{2 \lambda+1}+1-\vartheta_{2}}\left\|\left(\Delta \boldsymbol{u}_{h}, \nabla \partial_{3} \boldsymbol{u}\right)\right\|_{L^{2}}^{1+\vartheta_{2}}
\end{aligned}
$$


Plugging (20) and (23) into (19), we find

$$
\begin{aligned}
& \frac{\mathrm{d}}{\mathrm{d} t}\left[\left\|\nabla \boldsymbol{u}_{h}\right\|_{L^{2}}^{2}+\left\|\partial_{3} \boldsymbol{u}\right\|_{L^{2}}^{2}\right]+\left[\left\|\Delta \boldsymbol{u}_{h}\right\|_{L^{2}}^{2}+\left\|\nabla \partial_{3} \boldsymbol{u}\right\|_{L^{2}}^{2}\right] \\
& \leq C\left\|\partial_{3} \boldsymbol{u}\right\|_{L^{q}}\left\|\nabla \boldsymbol{u}_{h}\right\|_{L^{2}}^{\frac{2 q-3}{q}}\left\|\Delta \boldsymbol{u}_{h}\right\|_{L^{2}}^{\frac{3}{q}} \\
& \quad+C\left\|\left|u_{3}\right|^{\lambda}\right\|_{L^{2}}^{\frac{1-\vartheta_{1}}{\lambda}}\left\|\nabla\left(\left|u_{3}\right|^{\lambda}\right)\right\|_{L^{2}}^{\frac{2 \vartheta_{1}}{2 \lambda+1}}\left\|\partial_{3} \boldsymbol{u}\right\|_{L^{q}}^{\frac{\vartheta_{1}}{2 \lambda+1}+1-\vartheta_{2}}\left\|\left(\Delta \boldsymbol{u}_{h}, \nabla \partial_{3} \boldsymbol{u}\right)\right\|_{L^{2}}^{1+\vartheta_{2}} .
\end{aligned}
$$

Integrating in time and denoting by

$$
\begin{aligned}
\mathcal{J}^{2}(t) & =\sup _{\Gamma \leq \tau \leq t}\left[\left\|\nabla \boldsymbol{u}_{h}\right\|_{L^{2}}^{2}+\left\|\partial_{3} \boldsymbol{u}\right\|_{L^{2}}^{2}\right]+\int_{\Gamma}^{t}\left[\left\|\Delta \boldsymbol{u}_{h}\right\|_{L^{2}}^{2}+\left\|\nabla \partial_{3} \boldsymbol{u}\right\|_{L^{2}}^{2}\right] \mathrm{d} \tau \\
\mathcal{L}^{2}(t) & =\sup _{\Gamma \leq \tau \leq t}\left\|\left|u_{3}\right|^{\lambda}\right\|_{L^{2}}^{2}+\int_{\Gamma}^{t}\left\|\nabla\left(\left|u_{3}\right|^{\lambda}\right)\right\|_{L^{2}}^{2} \mathrm{~d} \tau, \quad \Gamma \leq t<\Gamma^{*}
\end{aligned}
$$

we deduce

$$
\begin{aligned}
& \mathcal{J}^{2}(t) \leq\left\|\nabla \boldsymbol{u}_{h}(\Gamma)\right\|_{L^{2}}^{2}+\left\|\partial_{3} \boldsymbol{u}(\Gamma)\right\|_{L^{2}}^{2}+C \mathcal{J}^{\frac{2 q-3}{q}}(t) \cdot \int_{\Gamma}^{t}\left\|\partial_{3} \boldsymbol{u}\right\|_{L^{q}}\left\|\Delta \boldsymbol{u}_{h}\right\|_{L^{2}}^{\frac{3}{q}} \mathrm{~d} \tau \\
& +C \mathcal{L}^{\frac{1-\vartheta_{1}}{\lambda}}(t) \cdot \int_{\Gamma}^{t}\left\|\nabla\left(\left|u_{3}\right|^{\lambda}\right)\right\|_{L^{2}}^{\frac{2 \vartheta_{1}}{2 \lambda+1}}\left\|\partial_{3} \boldsymbol{u}\right\|_{L^{q}}^{\frac{\vartheta_{1}}{2 \lambda+1}+1-\vartheta_{2}}\left\|\left(\Delta \boldsymbol{u}_{h}, \nabla \partial_{3} \boldsymbol{u}\right)\right\|_{L^{2}}^{1+\vartheta_{2}} \mathrm{~d} \tau \\
& \leq C+C \mathcal{J}^{\frac{2 q-3}{q}}(t) \cdot\left(\int_{\Gamma}^{t}\left\|\partial_{3} \boldsymbol{u}\right\|_{L^{q}}^{\frac{2 q}{2 q-3}} \mathrm{~d} \tau\right)^{\frac{2 q-3}{2 q}}\left(\int_{\Gamma}^{t}\left\|\Delta \boldsymbol{u}_{h}\right\|_{L^{2}}^{2} \mathrm{~d} \tau\right)^{\frac{3}{2 q}} \\
& +C \mathcal{L}^{\frac{1-\vartheta_{1}}{\lambda}}(t) \cdot\left(\int_{\Gamma}^{t}\left\|\nabla_{h}\left(\left|u_{3}\right|^{\lambda}\right)\right\|_{L^{2}}^{2} \mathrm{~d} \tau\right)^{\frac{\vartheta_{1}}{2 \lambda+1}} \\
& \cdot\left(\int_{\Gamma}^{t}\left\|\partial_{3} \boldsymbol{u}\right\|_{L^{q}}^{\frac{2 q}{2 q-3}} \mathrm{~d} \tau\right)^{\frac{2 q-3}{2 q}\left(\frac{\vartheta_{1}}{\lambda+1}+1-\vartheta_{2}\right) \mathrm{d} \tau} \cdot\left(\int_{\Gamma}^{t}\left\|\left(\Delta \boldsymbol{u}_{h}, \nabla \partial_{3} \boldsymbol{u}\right)\right\|_{L^{2}}^{2} \mathrm{~d} \tau\right)^{\frac{1+\vartheta_{2}}{2}} \\
& \leq C+C \tilde{\varepsilon} \mathcal{J}^{2}(t)+C \mathcal{L}^{\frac{1-\vartheta_{1}}{\lambda}}(t) \cdot \mathcal{L}^{\frac{2 \vartheta_{1}}{2 \lambda+1}}(t) \cdot \tilde{\varepsilon}^{\frac{\vartheta_{1}}{2 \lambda+1}+1-\vartheta_{2}} \cdot \mathcal{J}^{1+\vartheta_{2}}(t) \\
& \leq C+C \tilde{\varepsilon} \mathcal{J}^{2}(t)+C \tilde{\varepsilon}^{\frac{\vartheta_{1}}{2 \lambda+1}+1-\vartheta_{2}} \mathcal{J}^{1+\vartheta_{2}}(t) \mathcal{L}^{\frac{1-\vartheta_{1}}{\lambda}+\frac{2 \vartheta_{1}}{2 \lambda+1}}(t),
\end{aligned}
$$

where Hölder inequality with

$$
\frac{\vartheta_{1}}{2 \lambda+1}+\frac{2 q-3}{2 q}\left(\frac{\vartheta_{1}}{2 \lambda+1}+1-\vartheta_{2}\right)+\frac{1+\vartheta_{2}}{2}=1
$$

is used. 


\section{$2.2 L^{2 \lambda}$ estimate}

Taking the inner product of $(13)_{3}$ with $2 \lambda\left|u_{3}\right|^{2 \lambda-2} u_{3}$ in $L^{2}\left(\mathbb{R}^{3}\right)$, we get

$$
\frac{\mathrm{d}}{\mathrm{d} t}\left\|\left|u_{3}\right|^{\lambda}\right\|_{L^{2}}^{2}+C(\lambda)\left\|\nabla\left(\left|u_{3}\right|\right)^{\lambda}\right\|_{L^{2}}^{2}=2 \lambda \int_{\mathbb{R}^{3}} \partial_{3} \pi\left|u_{3}\right|^{2 \lambda-2} u_{3} \mathrm{~d} x \equiv L
$$

To process $L$, we derive from (16) that

$$
\begin{aligned}
-\triangle \partial_{3} \pi & =\sum_{i, j=1}^{3} \partial_{i} \partial_{j}\left(\partial_{3} u_{i} u_{j}+u_{i} \partial_{3} u_{j}\right)=2 \sum_{i, j=1}^{3} \partial_{i} \partial_{j}\left(u_{i} \partial_{3} u_{j}\right) \\
& =2 \sum_{i=1}^{2} \sum_{j=1}^{3} \partial_{i} \partial_{j}\left(u_{i} \partial_{3} u_{j}\right)+2 \sum_{j=1}^{3} \partial_{3} \partial_{j}\left(u_{3} \partial_{3} u_{j}\right) \\
\Rightarrow \partial_{3} \pi & =2 \sum_{i=1}^{2} \sum_{j=1}^{3} \mathcal{R}_{i} \mathcal{R}_{j}\left(u_{i} \partial_{3} u_{j}\right)+2 \sum_{j=1}^{3} \mathcal{R}_{3} \mathcal{R}_{j}\left(u_{3} \partial_{3} u_{j}\right) \equiv \pi_{1}+\pi_{2},
\end{aligned}
$$

where $\mathcal{R}_{i}=\frac{\partial_{i}}{\sqrt{-\triangle}}$ is the Riesz transformation, which is bounded from $\left.L^{r}\left(\mathbb{R}^{3}\right)\right)$ to itself for $1<r<\infty$.

In view of (29),

$$
\begin{aligned}
L & =2 \lambda \int_{\mathbb{R}^{3}}\left(\pi_{1}+\pi_{2}\right)\left|u_{3}\right|^{2 \lambda-2} u_{3} \mathrm{~d} x \\
& \leq C\left\|\boldsymbol{u}_{h}\right\|_{L^{c}}\left\|\partial_{3} \boldsymbol{u}\right\|_{L^{q}}\left\|u_{3}\right\|_{L^{d}}^{2 \lambda-1}+C\left\|u_{3}\right\|_{L^{\frac{2 \lambda q}{q-1}}\left\|\partial_{3} \boldsymbol{u}\right\|_{L^{q}}\left\|u_{3}\right\|_{L^{\frac{2 \lambda q}{q-1}}}^{2 \lambda-1},},
\end{aligned}
$$

where

$$
1 \leq c, d \leq \infty, \quad \frac{1}{c}+\frac{1}{q}+\frac{2 \lambda-1}{d}=1
$$

By Gagliardo-Nirenberg and interpolation inequalities,

$$
\begin{aligned}
L \leq & C\left\|\boldsymbol{u}_{h}\right\|_{L^{3 q}}^{1-\vartheta_{3}}\left\|\Delta \boldsymbol{u}_{h}\right\|_{L^{2}}^{\vartheta_{3}} \cdot\left\|\partial_{3} \boldsymbol{u}\right\|_{L^{q}} \cdot\left\|u_{3}\right\|_{L^{2 \lambda}}^{(2 \lambda-1)\left(1-\vartheta_{4}\right)}\left\|u_{3}\right\|_{L^{(2 \lambda+1) q}}^{(2 \lambda-1) \vartheta_{4}} \\
& +C\left\|\partial_{3} \boldsymbol{u}\right\|_{L^{q}}\left\|\left|u_{3}\right|^{\lambda}\right\|_{L^{\frac{2 q}{q-1}}}^{2}
\end{aligned}
$$

where

$$
\frac{1}{c}=\left(1-\vartheta_{3}\right) \frac{1}{3 q}+\vartheta_{3}\left(-\frac{2}{3}+\frac{1}{2}\right), \quad \frac{1}{d}=\frac{1-\vartheta_{4}}{2 \lambda}+\frac{\vartheta_{4}}{(2 \lambda+1) q}, \quad 0 \leq \vartheta_{3}, \vartheta_{4} \leq 1 .
$$


By (6) and Lemma 3,

$$
\begin{aligned}
L \leq & C\left\|\nabla \boldsymbol{u}_{h}\right\|_{L^{2}}^{\frac{2\left(1-\vartheta_{3}\right)}{3}}\left\|\partial_{3} \boldsymbol{u}\right\|_{L^{q}}^{\frac{1-\vartheta_{3}}{3}} \cdot\left\|\Delta \boldsymbol{u}_{h}\right\|_{L^{2}}^{\vartheta_{3}}\left\|\partial_{3} \boldsymbol{u}\right\|_{L^{q}}\left\|u_{3}\right\|_{L^{2 \lambda}}^{(2 \lambda-1)\left(1-\vartheta_{4}\right)} \\
& \cdot\left\|\nabla\left|u_{3}\right|^{\lambda}\right\|_{L^{2}}^{\frac{2(2 \lambda-1) \vartheta_{4}}{2 \lambda+1}}\left\|\partial_{3} u_{3}\right\|_{L^{q}}^{\frac{(2 \lambda-1) \vartheta_{4}}{2 \lambda+1}}+C\left\|\partial_{3} \boldsymbol{u}\right\|_{L^{q}} \cdot\left\|\left|u_{3}\right|^{\lambda}\right\|_{L^{2}}^{\frac{2 q-3}{q}}\left\|\nabla\left(\left|u_{3}\right|^{\lambda}\right)\right\|_{L^{2}}^{\frac{3}{q}} \\
\leq & C\left\|\nabla \boldsymbol{u}_{h}\right\|_{L^{2}}^{\frac{2\left(1-\vartheta_{3}\right)}{3}}\left\|\partial_{3} \boldsymbol{u}\right\|_{L^{q}}^{\frac{1-\vartheta_{3}}{3}+1+\frac{(2 \lambda-1) \vartheta_{4}}{2 \lambda+1}}\left\|\Delta \boldsymbol{u}_{h}\right\|_{L^{2}}^{\vartheta_{3}}\left\|\left|u_{3}\right|^{\lambda}\right\|_{L^{2}}^{\frac{(2 \lambda-1)\left(1-\vartheta_{4}\right)}{\lambda}} \\
& \cdot\left\|\nabla\left(\left|u_{3}\right|^{\lambda}\right)\right\|_{L^{2}}^{\frac{2(2 \lambda-1) \vartheta_{4}}{2 \lambda+1}}+C\left\|\partial_{3} \boldsymbol{u}\right\|_{L^{q}} \cdot\left\|\left|u_{3}\right|^{\lambda}\right\|_{L^{2}}^{\frac{2 q-3}{q}}\left\|\nabla\left(\left|u_{3}\right|^{\lambda}\right)\right\|_{L^{2}}^{\frac{3}{q}}
\end{aligned}
$$

Putting (32) into (28), and integrating in time yields

$$
\begin{aligned}
\mathcal{L}^{2}(t) \leq & C+C \mathcal{J}^{\frac{2\left(1-\vartheta_{3}\right)}{3}}(t) \mathcal{L}^{\frac{(2 \lambda-1)\left(1-\vartheta_{4}\right)}{\lambda}}(t) \cdot \int_{\Gamma}^{t}\left\|\partial_{3} \boldsymbol{u}\right\|_{L^{q}}^{\frac{1-\vartheta_{3}}{q}+1+\frac{(2 \lambda-1) \vartheta_{4}}{2 \lambda+1}}\left\|\Delta \boldsymbol{u}_{h}\right\|_{L^{2}}^{\vartheta_{3}} \\
& \left\|\nabla\left(\left|u_{3}\right|^{\lambda}\right)\right\|_{L^{2}}^{\frac{2(2 \lambda-1) \vartheta_{4}}{2 \lambda+1}} \mathrm{~d} \tau+C \mathcal{L}^{\frac{2 q-3}{q}}(t) \cdot \int_{\Gamma}^{t}\left\|\partial_{3} \boldsymbol{u}\right\|_{L^{q}}\left\|\nabla\left(\left|u_{3}\right|^{\lambda}\right)\right\|_{L^{2}}^{\frac{3}{q}} \mathrm{~d} \tau \\
\leq & C+C \tilde{\varepsilon}^{\frac{1-\vartheta_{3}}{3}+1+\frac{(2 \lambda-1) \vartheta_{4}}{2 \lambda+1}} \mathcal{J}^{\frac{2\left(1-\vartheta_{3}\right)}{3}+\vartheta_{3}}(t) \mathcal{L}^{\frac{(2 \lambda-1)\left(1-\vartheta_{4}\right)}{\lambda}+\frac{2(2 \lambda-1) \vartheta_{4}}{2 \lambda+1}}(t)+C \tilde{\varepsilon} \mathcal{L}^{2}(t),
\end{aligned}
$$

where just as in (26), Hölder inequality with

$$
\frac{2 q-3}{2 q}\left[\frac{1-\vartheta_{3}}{3}+1+\frac{(2 \lambda-1) \vartheta_{4}}{2 \lambda+1}\right]+\frac{\vartheta_{3}}{2}+\frac{(2 \lambda-1) \vartheta_{4}}{2 \lambda+1}=1
$$

is applied.

\subsection{Closing estimate}

By (26) and (33), we have

$$
\begin{aligned}
\mathcal{J}^{2}(t) & \leq C+C \tilde{\varepsilon} \mathcal{J}^{2}(t)+C \tilde{\varepsilon}^{\frac{\vartheta_{1}}{2 \lambda+1}+1-\vartheta_{2}} \mathcal{J}^{j_{1}}(t) \mathcal{L}^{l_{1}}(t) \\
\mathcal{L}^{2}(t) & \leq C+C \tilde{\varepsilon}^{\frac{1-\vartheta_{3}}{3}+1+\frac{(2 \lambda-1) \vartheta_{4}}{2 \lambda+1}} \mathcal{J}^{j_{2}}(t) \mathcal{L}^{l_{2}}(t)+C \tilde{\varepsilon} \mathcal{L}^{2}(t)
\end{aligned}
$$

where

$$
\begin{array}{rlrl}
j_{1} & =1+\vartheta_{2}, & l_{1} & =\frac{1-\vartheta_{1}}{\lambda}+\frac{2 \vartheta_{1}}{2 \lambda+1}, \\
j_{2}=\frac{2\left(1-\vartheta_{3}\right)}{3}+\vartheta_{3}, & l_{2}=\frac{(2 \lambda-1)\left(1-\vartheta_{4}\right)}{\lambda}+\frac{2(2 \lambda-1) \vartheta_{4}}{2 \lambda+1}
\end{array}
$$


and $0 \leq \vartheta_{i} \leq 1(1 \leq i \leq 4)$ should satisfy

$$
\begin{aligned}
& {\left[\frac{1-\vartheta_{1}}{2 \lambda}+\frac{\vartheta_{1}}{(2 \lambda+1) q}\right]+\left[\frac{1-\vartheta_{2}}{q}+\vartheta_{2}\left(-\frac{1}{3}+\frac{1}{2}\right)\right]=\frac{1}{2}} \\
& \frac{\vartheta_{1}}{2 \lambda+1}+\frac{2 q-3}{2 q}\left(\frac{\vartheta_{1}}{2 \lambda+1}+1-\vartheta_{2}\right)+\frac{1+\vartheta_{2}}{2}=1 \\
& {\left[\left(1-\vartheta_{3}\right) \frac{1}{3 q}+\vartheta_{3}\left(-\frac{2}{3}+\frac{1}{2}\right)\right]+\frac{1}{q}+(2 \lambda-1)\left[\frac{1-\vartheta_{4}}{2 \lambda}+\frac{\vartheta_{4}}{(2 \lambda+1) q}\right]=1,} \\
& \frac{2 q-3}{2 q}\left[\frac{1-\vartheta_{3}}{3}+1+\frac{(2 \lambda-1) \vartheta_{4}}{2 \lambda+1}\right]+\frac{\vartheta_{3}}{2}+\frac{(2 \lambda-1) \vartheta_{4}}{2 \lambda+1}=1
\end{aligned}
$$

in view of (21), (22), (27), (30), (31) and (34).

After tedious calculations, we can solve (38) as

$$
\begin{aligned}
& \vartheta_{1}=\frac{(2 \lambda-3)(2 \lambda+1)(3-q)}{2 \lambda q+3 q+3 \lambda-9}, \quad \vartheta_{2}=3 \frac{\lambda(3-2 q)+(5 q-6)}{2 \lambda q+3 q+3 \lambda-9}, \\
& \vartheta_{3}=\frac{4 \lambda(q+1)-(10 q+3)}{2 \lambda q-q+\lambda-3}, \quad \vartheta_{4}=\frac{(2 \lambda+1)[9+(3-2 \lambda) q]}{3(2 \lambda-1)(2 \lambda q-q+\lambda-3)} .
\end{aligned}
$$

Reducing $0 \leq \vartheta_{i} \leq 1(i=1,2,3,4)$ yields

$$
\begin{gathered}
\text { if } \left.\lambda \in\left[\frac{19}{10}, \frac{33}{16}\right]\right] \quad\left[\frac{33}{16}, \frac{25+2 \sqrt{37}}{18}\right]\left[\frac{25+2 \sqrt{37}}{18}, \frac{5}{2}\right] \\
\text { then } q \in\left[\frac{39-6 \lambda}{16(\lambda-1)}, \frac{4 \lambda-3}{10-4 \lambda}\right]\left[\frac{39-6 \lambda}{16(\lambda-1)}, 3\right]\left[\frac{5}{2}, 3\right] \\
{\left[\frac{3(4 \lambda-5)}{2(2 \lambda-1)}, 3\right]\left[\frac{3 \lambda}{9-2 \lambda}, 3\right]}
\end{gathered}
$$

respectively. Consequently, if

$$
\lambda=\lambda_{0} \equiv \frac{25+2 \sqrt{37}}{18} \approx 2.06475,
$$

the range of $q$ is the largest one:

$$
q \in\left[\frac{3 \sqrt{37}}{4}-3,3\right]
$$

By (39), (37) becomes

$$
\begin{array}{ll}
j_{1}=\frac{4 \lambda(3-q)+9(2 q-3)}{2 \lambda q+3 q+3 \lambda-9}, & l_{1}=\frac{4 q-3}{2 \lambda q+3 q+3 \lambda-9}, \\
j_{2}=\frac{(2 \lambda-3)(4 q+3)}{3(2 \lambda q-q+\lambda-3)}, & l_{2}=\frac{21+10 q-6 \lambda(2 q+1)}{3(2 \lambda q-q+\lambda-3)} .
\end{array}
$$


When (41) and (42) holds, it is obvious that $1 \leq j_{1}<2$, and we may apply Hölder inequality to (35),

$$
\begin{aligned}
\mathcal{J}^{2}(t) & \leq C+C \tilde{\varepsilon} \mathcal{J}^{2}(t)+\frac{1}{2} \mathcal{J}^{2}(t)+C \mathcal{L}^{\frac{2 l_{1}}{2-j_{1}}}(t) \\
& \leq C+C \tilde{\varepsilon} \mathcal{J}^{2}(t)+\frac{1}{2} \mathcal{J}^{2}(t)++C \mathcal{L}^{\frac{2}{2 \lambda-3}}(t)
\end{aligned}
$$

Now choose $0<\tilde{\varepsilon} \ll 1$ sufficiently small such that

$$
C \tilde{\varepsilon} \leq \frac{1}{4}
$$

we have

$$
\mathcal{J}(t) \leq C+C \mathcal{L}^{\frac{1}{2 \lambda-3}}(t)
$$

Plugging (46) into (36), and choosing $\tilde{\varepsilon}$ such that

$$
C \tilde{\varepsilon}^{\frac{1-\vartheta_{3}}{3}+1+\frac{(2 \lambda-1) \vartheta_{4}}{2 \lambda+1}} \leq \frac{1}{4}
$$

besides (45), we find

$$
\mathcal{L}^{2}(t) \leq C+\frac{1}{4} \mathcal{L} \frac{1}{2 \lambda-3} j_{2}+l_{2}(t)+\frac{1}{2} \mathcal{L}^{2}(t)=C+\frac{3}{4} \mathcal{L}^{2}(t) \Rightarrow \mathcal{L}^{2}(t) \leq C .
$$

Combining (46) and (48), we see that $\left\|\nabla \boldsymbol{u}_{h}(t)\right\|_{L^{2}}$ is uniformly bounded on $t \in$ $\left[\Gamma, \Gamma^{*}\right)$ as desired. The proof of Theorem 2 is completed.

Remark 4 Cao [2] took $\lambda=2$ to deduce (8), which corresponds to the range of $q$ in case $\lambda=2$ in (40). In our paper, we treat all the possibilities to make the range of $q$ as large as possible. The method involves a generalized multiplicative Sobolev inequality (see Lemma 3 ) and the general $L^{2 \lambda}$ estimate, but not just $L^{4}$ estimate. For some applications of general $L^{2 \lambda}$ estimates, we refer to [10,32], which improves [5].

Acknowledgements This work is supported by the National Natural Science Foundation of China (Grant No. 11501125).

Open Access This article is distributed under the terms of the Creative Commons Attribution 4.0 International License (http://creativecommons.org/licenses/by/4.0/), which permits unrestricted use, distribution, and reproduction in any medium, provided you give appropriate credit to the original author(s) and the source, provide a link to the Creative Commons license, and indicate if changes were made.

\section{References}

1. Beirão da Veiga, H.: A new regularity class for the Navier-Stokes equations in $\mathbb{R}^{n}$. Chin. Ann. Math. Ser. B 16, 407-412 (1995) 
2. Cao, C.S.: Sufficient conditions for the regularity to the 3D Navier-Stokes equations. Discrete Contin. Dyn. Syst. 26, 1141-1151 (2010)

3. Cao, C.S., Titi, E.S.: Regularity criteria for the three-dimensional Navier-Stokes equations. Indiana Univ. Math. J. 57, 2643-2661 (2008)

4. Cao, C.S., Titi, E.S.: Global regularity criterion for the 3D Navier-Stokes equations involving one entry of the velocity gradient tensor. Arch. Ration. Mech. Anal. 202, 919-932 (2011)

5. Cao, C.S., Wu, J.H.: Two regularity criteria for the 3D MHD equations. J. Differ. Equ. 248, 2263-2274 (2010)

6. Chemin, J.Y., Zhang, P.: On the critical one component regularity for the 3-D Navier-Stokes equations. arXiv:1310.6442 [math.AP] 24 Oct (2013)

7. Constantin, P., Fioas, C.: Navier-Stokes equations. Chicago Lectures in Mathematics Series (1988)

8. Eskauriaza, L., Serëgin, G.A., Šverák, V.: $L_{3, \infty}$-solutions of Navier-Stokes equations and backward uniqueness. Russ. Math. Surv. 58, 211-250 (2003)

9. Hopf, E.: Über die Anfangwertaufgaben für die hydromischen Grundgleichungen. Math. Nachr. 4, 213-321 (1951)

10. Jia, X.J., Zhou, Y.: A new regularity criterion for the 3D incompressible MHD equations in terms of one component of the gradient of pressure. J. Math. Anal. Appl. 396, 345-350 (2012)

11. Jia, X.J., Zhou, Y.: Remarks on regularity criteria for the Navier-Stokes equations via one velocity component. Nonlinear Anal. Real World Appl. 15, 239-245 (2014)

12. Jia, X.J., Zhou, Y.: Ladyzhenskaya-Prodi-Serrin type regularity criteria for the 3D incompressible MHD equations in terms of $3 \times 3$ mixture matrices. Nonlinearity 28, 3289-3307 (2015)

13. Jia, X.J., Zhou, Y.: Regularity criteria for the 3D MHD equations via partial derivatives. II. Kinet. Relat. Models 7, 291-304 (2014)

14. Jia, X.J., Zhou, Y.: Regularity criteria for the 3D MHD equations via partial derivatives. Kinet. Relat. Models 5, 505-516 (2012)

15. Jia, X.J., Zhou, Y.: Regularity criteria for the 3D MHD equations involving partial components. Nonlinear Anal. Real World Appl. 13, 410-418 (2012)

16. Kukavica, I., Ziane, M.: One component regularity for the Navier-Stokes equations. Nonlinearity $\mathbf{1 9}$, 453-469 (2006)

17. Kukavica, I., Ziane, M.: Navier-Stokes equations with regularity in one direction. J. Math. Phys. 48, 065203 (2007)

18. Ladyzhenskaya, O.A.: The mathematical theory of viscous incompressible flow, Moscow (1970)

19. Leray, J.: Sur le mouvement d'un liquide visqueux emplissant l'espace. Acta Math. 63, 193-248 (1934)

20. Neustupa, J., Novotný, A., Penel, P.: An interior regularity of a weak solution to the Navier-Stokes equations in dependence on one component of velocity, topics in mathematical fluid mechanics. Quad. Mat. 10, 163-183 (2002)

21. Penel, P., Pokorný, M.: On anisotropic regularity criteria for the solutions to 3D Navier-Stokes equations. J. Math. Fluid Mech. 13, 341-353 (2011)

22. Penel, P., Pokorný, M.: Some new regularity criteria for the Navier-Stokes equations containg gradient of the velocity. Appl. Math. 49, 483-493 (2004)

23. Pokorný, M.: On the result of He concerning the smoothness of solutions to the Navier-Stokese equations. Electron. J. Differ. Equ. 2003, 1-8 (2003)

24. Prodi, G.: Un teorema di unicitá per le equazioni di Navier-Stokes. Ann. Mat. Pura Appl. 48, 173-182 (1959)

25. Lemarié-Rieusset, P.G.: Recent Developments in the Navier-Stokes Problem. Chapman and Hall, London (2002)

26. Serrin, J.: On the interior regularity of weak solutions of the Navier-Stokes equations. Arch. Ration. Mech. Anal. 9, 187-195 (1962)

27. Skalak, Z.: On the regularity of the solutions to the Navier-Stokes equations via the gradient of one velocity component. Nonlinear Anal. 104, 84-89 (2014)

28. Temam, R.: Navier-Stokes Equations, Theory and Numercial Analysis. AMS Chelsea Publishing, Chelsea (2001)

29. Zhang, Z.J.: A Serrin-type reuglarity criterion for the Navier-Stokes equations via one velocity component. Commun. Pure Appl. Anal. 12, 117-124 (2013)

30. Zhang, Z.J.: A logarithmically improved regularity criterion for the 3D MHD system involving the velocity field in homogeneous Besov spaces. Ann. Pol. Math. 118, 51-57 (2016) 
31. Zhang, Z.J., Alzahrani, F., Hayat, T., Zhou, Y.: Two new regularity criteria for the NavierCStokes equations via two entries of the velocity Hessian tensor. Appl. Math. Lett. 37, 124-130 (2014)

32. Zhang, Z.J., Li, P., Yu, G.H.: Regularity criteria for the 3D MHD equations via one directional derivative of the pressure. J. Math. Anal. Appl. 401, 66-71 (2013)

33. Zhang, Z.J., Yao, Z.A., Lu, M., Ni, L.D.: Some Serrin-type regularity criteria for weak solutions to the Navier-Stokese equations. J. Math. Phys. 52, 053103 (2011)

34. Zhou, Y.: A new regularity criterion for weak solutions to the Navier-Stokes equations. J. Math. Pures Appl. 84, 1496-1514 (2005)

35. Zhou, Y.: A new regularity criterion for the Navier-Stokes equations in terms of the gradient of one velocity component. Methods Appl. Anal. 9, 563-578 (2002)

36. Zhou, Y., Pokorný, M.: On the regularity of the solutions of the Navier-Stokes equations via one velocity component. Nonlinearity 23, 1097-1107 (2010)

37. Zhou, Y., Pokorný, M.: On a regularity criterion for the Navier-Stokes equations involving gradient of one velocity component. J. Math. Phys. 50, 123514 (2009) 